\title{
EXISTENCE AND UNIQUENESS RESULTS FOR CERTAIN FRACTIONAL BOUNDARY VALUE PROBLEMS
}

\author{
Dilsher N. AbDulqader and Shayma Adil Murad \\ Dept. of Mathematics, College of Science, University of Duhok, Kurdistan Region-Iraq.
}

(Received: May 26, 2019; Accepted for Publication: September 19, 2019)

\begin{abstract}
In this study, by applying some fixed point theorems when $\alpha \in(n-1, n]$, the existence and uniqueness theorem for certain fractional differential equations with fractional boundary conditions is established. The theories are illustrated by examples.
\end{abstract}

KEY WORDS: Existence theorem, Riemann-Liouville fractional derivative, Riemann-Liouville fractional integral.

\section{INTRODUCTION}

$\mathbf{I}^{\mathrm{n}}$ $n$ the last decades the fractional calculus was popular and played an important role in different fields of science especially chemistry, fluid mechanics, elasticity, heat conduction in materials with memory, physics and engineering, see [10-11]. The subject of this work is related to the differential equation involving non-integer derivative. Many authors discussed the fractional order of differentiation and it was found that the fractional order of derivatives and integrals is just tangible as that of integer order. Thus the fractional derivative is an extension of the familiar derivative $d^{n} f(t) / d t^{n}$, to non-integer values of $n$, see $[3,6,8]$. Recently several researchers deals with fixed point theorems to discuss the existence and uniqueness of solution for fractional differential equations with initial or boundary conditions; for more details see [4,5,12,14-16]. Shuman et al [17] discussed the uniqueness of the solution to a class of differential systems with coupled integral boundary conditions under the Lipschitz condition. The solution of the existence and uniqueness and the solution of nonexistence of positive solution are obtained by means of the iterative technique in [18]. Rian Yan et al [13] used Banach's fixed point theorem and Schaefer's fixed point theorem to establish some criteria of existence for the boundary problems with non-local boundary condition involving the Caputo fractional derivative. The existence of solution for the ordinary differential equation of non-integer order through the method of fixed point in the large has been discussed in [2]. The purpose of this work is to prove the existence and uniqueness through Krasnoselskii fixed point theorem for the problem

$$
\begin{aligned}
& D^{\alpha} y(t)=f(t, y), n-1<\alpha \leq n,(1) \\
& \beta_{r} D^{(\alpha-r)} y(a)+\gamma_{r} D^{(\alpha-r)} y(T)=C_{r}, \\
& \text { where } \quad r=1,2,3, \ldots, n,
\end{aligned}
$$

where $D^{\alpha}$ is the Riemann-Liouville fractional derivative, $t=[a, T]$, and $a, T, \beta_{r}, \gamma_{r}$ and $C_{r}$ are constants, We consider the space $C([a, T], R)$ to be the Banach space of all continuous functions defined from $[a, T]$ into $\mathrm{R}$, with the norm $\|y\|=\sup \{|y(t)|: t \in I\}$.

The result is more general and contains uniqueness solution. We verify the result by contracting an interesting example. 


\section{PRELIMINARIES}

Let us give some definitions, theorems and lemmas that are basic and needed at various places in this work. For references see $[3,6,7,11]$.

Definition 2.1 Let $f$ be a function wih is defined almost everywhere (a.e) on [a,b], for $\alpha>0$, we define:

where $\Gamma(\alpha)$ is the Gamma function.

$$
{ }_{a}^{b} D^{-\alpha} f=\frac{1}{\Gamma(\alpha)} \int_{a}^{b}(b-s)^{\alpha-1} f(s) d s,
$$

Definition 2.2 The Riemann-Liouville fractional derivative of order $\alpha>0$ for a function $y(t)$ is defined by

$$
{ }_{a}^{t} D^{\alpha} y(t)=\frac{1}{\Gamma(n-\alpha)}\left(\frac{d}{d t}\right)^{n} \int_{a}^{t}(t-s)^{n-\alpha-1} y(s) d s \quad n-1<\alpha \leq n,
$$

where $n=[\alpha]+1$ and $[\alpha]$ denotes the integer part of $\alpha$.

Lemma 2.3 If $\alpha>0$; $\mathrm{n}$ is the smallest integer $>\alpha$; $\mathrm{f}(\mathrm{x})$ is in $\mathrm{L}(\mathrm{a}, \mathrm{b})$ and ${ }_{a}^{t} I^{1-\alpha} f$ exists and absolutely continuous on $[\mathrm{a}, \mathrm{b}]$, then ${ }_{a}^{a} I^{i-\alpha} f=k_{i}$ exists for $i=1,2, \ldots, n ;{ }_{a}^{t} I^{\alpha} f$ exists almost everywhere on $[a, b]$, is in $L(a, b)$ and

$$
{ }_{a}^{t} I^{\alpha}{ }_{a}^{t} I^{-\alpha} f(t)=f(t)-\sum_{i=1}^{n} \frac{k_{i}(t-a)^{\alpha-i}}{\Gamma(\alpha-i+1)} \quad \text { a.e. on } \quad a \leq t \leq b .
$$

Furthermore, the inequality works everywhere on $(a, b]$, if in additional, $f(x)$ is continuous on (a,b].

Lemma 2.4 Let $f(t) \in L_{1}[a, T]$ and $\alpha, \beta \geq 0$. Then

$$
I^{\alpha} I^{\beta} f(t)=I^{\alpha+\beta} f(t)=I^{\beta} I^{\alpha} f(t)
$$

is defined almost everywhere on $[\mathrm{a}, \mathrm{T}]$. Moreover, if $f(t) \in C[a, T]$, then the identity (3) is true for all $t \in[a, T]$

Lemma 2.5 Let $\alpha>0$, that $f(t) \in C([a, T])$, then $D^{\alpha} I^{\alpha} f(t)=f(t)$ for all $t \in[a, T]$.

Lemma 2.6 Let $\alpha, \beta \in R, \beta>-1$. If $t>a$, then

$$
D^{-\alpha} \frac{(t-a)^{\beta}}{\Gamma(\beta+1)} \begin{cases}\frac{(t-a)^{\alpha+\beta}}{\Gamma(\alpha+\beta+1)} & ; \alpha+\beta \neq \text { negative integer } \\ 0 & ; \alpha+\beta=\text { negative integer }\end{cases}
$$

Lemma 2.7 If $\alpha>0$ and $f(x)$ is Lebesgue integrable function on [a,b], then

$$
{ }_{a}^{t} I^{-\alpha}{ }_{a}^{s} I^{\alpha} f=f(x) \text { a.e. for all } t \in[a, b]
$$


Theorem 2.8 ("Krasnosel'skiu fixed point teorem"). Let M be a closed-convex bounde nonempty subset of a Banach space X. Let $A$ and $B$ be two operators such that

(1) $A x+B y=M$, whenever $x, y \in M$,

(2) A is compact and continuous,

(3) B is a contraction mapping.

Then there exists $z \in M$ such that $z=A z+B z$.

Lemma 2.9 Let $y(t) \in C([a, T])$ and $n-1<\alpha \leq n$, then the unique solution of the fractional boundary value problem (1)-(2) is given by

$$
y(t)={ }_{a}^{t} I^{\alpha} f(t, y)+\sum_{i=1}^{n} \frac{k_{i}(t-a)^{\alpha-i}}{\Gamma(\alpha-i+1)}
$$

Where

$$
k_{i}=\frac{1}{\beta_{i}+\gamma_{i}}\left[C_{i}-\gamma_{i} \quad{ }_{a}^{T} I^{i} f(t, y)-\gamma_{i} \sum_{m=1}^{i-1} \frac{k_{m}(T-a)^{i-m}}{\Gamma(i-m+1)}\right]
$$

Proof: By applying the lemma (2.3), we may reduce (1) to an equivalent equation

$$
y(t)={ }_{a}^{t} I^{\alpha} f(t, y)+\sum_{i=1}^{n} \frac{k_{i}(t-a)^{\alpha-i}}{\Gamma(\alpha-i+1)}
$$

For $k_{i} \in R$. From the boundary condition (2), it follows

$$
y^{(\alpha-r)}(t)={ }_{a}^{t} I^{r-\alpha}{ }_{a}^{t} I^{\alpha} f(t, y)+\sum_{i=1}^{n} \frac{k_{i}{ }_{a}^{t} I^{r-\alpha}(t-a)^{\alpha-i}}{\Gamma(\alpha-i+1)},
$$

by using the lemma (2.7) and lemma (2.9), we arrive at

$$
y^{(\alpha-r)}(t)={ }_{a}^{t} I^{r} f(t, y)+\sum_{i=1}^{n} \frac{k_{i}(t-a)^{r-i}}{\Gamma(r-i+1)}
$$

when $r \geq i, r=1,2,3, \ldots, n$, now to find the values of $k_{i}$ applying the boundary conditions (2), we find

$$
y^{(\alpha-r)}(a)=k_{r}, \quad y^{(\alpha-r)}(T)={ }_{a}^{T} I^{r} f(t, y)+\sum_{i=1}^{n} \frac{k_{i}(T-a)^{r-i}}{\Gamma(r-i+1)} .
$$

Substituting (5) in (2), we obtain

$$
C_{r}=\beta_{r} k_{r}+\gamma_{r} \quad{ }_{a}^{T} I^{r} f(t, y)+\gamma_{r} \sum_{i=1}^{n} \frac{k_{i}(T-a)^{r-i}}{\Gamma(r-i+1)}
$$

when $n=1$ and $r=1$, we have

$$
\beta_{1} k_{1}+\gamma_{1}{ }_{a}^{T} I^{1} f(t, y)+\gamma_{1} k_{1}=C_{1} \Rightarrow k_{1}=\frac{1}{\left(\beta_{1}+\gamma_{1}\right)}\left[C_{1}-\gamma_{1} \quad{ }_{a}^{T} I^{1} f(t, y)\right],
$$

when $n=2$ and $r=2$, we have

when $n=3$ and $r=3$, we have

$$
k_{2}=\frac{1}{\left(\beta_{2}+\gamma_{2}\right)}\left[C_{2}-\gamma_{2} \quad{ }_{a}^{T} I^{2} f(t, y)-\gamma_{2} k_{1} \frac{(T-a)}{\Gamma(2)}\right],
$$

when $r=n$, we obtain

$$
k_{3}=\frac{1}{\left(\beta_{3}+\gamma_{3}\right)}\left[C_{3}-\gamma_{3} \quad{ }_{a}^{T} I^{3} f(t, y)-\gamma_{3} k_{1} \frac{(T-a)^{2}}{\Gamma(3)}-\gamma_{3} k_{2} \frac{(T-a)}{\Gamma(2)}\right],
$$


then the solution of (1)-(2) is

$$
k_{n}=\frac{1}{\left(\beta_{n}+\gamma_{n}\right)}\left[C_{n}-\gamma_{n}{ }_{a}^{T} I^{n} f(t, y)-\gamma_{n} \sum_{i=1}^{n-1} \frac{k_{i}(T-a)^{r-i}}{\Gamma(n-i+1)}\right],
$$

$$
y(t)=\sum_{i=1}^{n} \frac{k_{i}(t-a)^{\alpha-i}}{\Gamma(\alpha-i+1)}+\frac{1}{\Gamma(\alpha)} \int_{a}^{t}(t-s)^{\alpha-1} f(t, y(s)) d s,
$$

Where

$$
k_{i}=\frac{1}{\beta_{i}+\gamma_{i}}\left[C_{i}-\frac{\gamma_{i}}{\Gamma(i)} \int_{a}^{T}(T-s)^{i-1} f(t, y(s)) d s-\gamma_{i} \sum_{m=1}^{i-1} \frac{k_{m}(T-a)^{i-m}}{\Gamma(i-m+1)}\right]
$$

Next, we need to prove that $y(t)$ satisfies the differential equation(1) a.e.

From equation (6) and Definition (2.1), we have

$$
y(t)=\sum_{i=1}^{n} \frac{k_{i}(t-a)^{\alpha-i}}{\Gamma(\alpha-i+1)}+{ }_{a}^{t} I^{\alpha} f
$$

From (7), we obtain

$$
{ }_{a}^{t} I^{-\alpha} y(t)=\sum_{i=1}^{n}{ }_{a}^{t} I^{-\alpha} \frac{k_{i}(s-a)^{\alpha-i}}{\Gamma(\alpha-i+1)}+{ }_{a}^{t} I^{-\alpha}{ }_{a}^{s} I^{\alpha} f
$$

From Lemmas (2.6) and (2.7), we have

$$
\sum_{i=1}^{n}{ }_{a}^{t} I^{-\alpha} \frac{k_{i}(s-a)^{\alpha-i}}{\Gamma(\alpha-i+1)}=0 \text { and }{ }_{a}^{t} I^{-\alpha}{ }_{a}^{s} I^{\alpha} f=f(t, y) \quad \text { a.e. forall } t \in[a, b]
$$

Hence the equation (6), becomes

$$
{ }_{a}^{t} I^{-\alpha} y(t)=f(t, y) \quad \text { a.s } \quad \text { on } \quad(a, b)
$$

Next, we need prove that $y(t)$ satisfies the equation (2), from (7), we obtain

$$
{ }_{a}^{t} I^{r-\alpha} y(t)=\sum_{i=1}^{n}{ }_{a}^{t} I^{r-\alpha} \frac{k_{i}(s-a)^{\alpha-i}}{\Gamma(\alpha-i+1)}+{ }_{a}^{t} I^{r-\alpha}{ }_{a}^{s} I^{\alpha} f,
$$

by using the Lemma (2.6), we have

$$
D^{\alpha-r} y(t)=\sum_{i=1}^{n} \frac{k_{i}(s-a)^{r-i}}{\Gamma(r-i+1)}+{ }_{a}^{s} I^{r} f
$$

by applying the boundary condition (2), we find

$$
\beta_{r} D^{(\alpha-r)} y(a)+\gamma_{r} D^{(\alpha-r)} y(T)=\beta_{r} k_{r}+\gamma_{r} \quad{ }_{a}^{T} I^{r} f(t, y)+\gamma_{r} \sum_{i=1}^{n} \frac{k_{i}(T-a)^{r-i}}{\Gamma(r-i+1)}
$$

Now, when $0<\alpha \leq 1, \mathrm{r}=1$ and $k_{1}=\frac{1}{\beta_{1}+\gamma_{1}}\left[C_{1}-\gamma_{1}{ }_{a}^{T} I^{1} f(t, y)\right]$ then we obtain

$$
\beta_{1} D^{(\alpha-1)} y(a)+\gamma_{1} D^{(\alpha-1)} y(T)=\left(\beta_{1}+\gamma_{1}\right) k_{1}+\gamma_{1} \quad{ }_{a}^{T} I^{1} f(t, y)=C_{1},
$$


when $1<\alpha \leq 2, \mathrm{r}=1,2$ and $k_{2}=\frac{1}{\beta_{2}+\gamma_{2}}\left[C_{2}-\gamma_{2} \quad{ }_{a}^{T} I^{2} f(t, y)-\gamma_{2} k_{1}(T-a)\right]$,

we have two boundary conditions, the first one is satisfied from equation (10) and the second condition it follows

$$
\beta_{2} D^{(\alpha-2)} y(a)+\gamma_{2} D^{(\alpha-2)} y(T)=\left(\beta_{2}+\gamma_{2}\right) k_{2}+\gamma_{2} \quad{ }_{a}^{T} I^{2} f(t, y)+\gamma_{2} k_{1}(T-a)=C_{2},
$$

and using the same way, for $n-1<\alpha \leq n, \mathrm{r}=1,2,3, \ldots, \mathrm{n}$. we find

$$
\beta_{n} D^{(\alpha-n)} y(a)+\gamma_{n} D^{(\alpha-n)} y(T)=\beta_{n} k_{n}+\gamma_{n} \quad{ }_{a}^{T} I^{n} f(t, y)+\gamma_{n} \sum_{i=1}^{n} \frac{k_{n}(T-a)^{n-i}}{\Gamma(n-i+1)}=C_{n} .
$$

Then the equation (4) satisfies the boundary value problem (1)-(2).

\section{Main Results}

In this section we establish the existence and uniqueness theorem of the boundary value problem (1)(2).

\section{Theorem 3.1 Assume that}

(H1) There exists a constant $M>0$, such that: $|f(t, y)| \leq M$ for each $t \in I$ and all $y \in R$.

(H2) There exists a constant $Q>0$, such that: $\left|f\left(t, y_{1}\right)-f\left(t, y_{2}\right)\right| \leq Q\left|y_{1}-y_{2}\right|$, for each $y_{1}, y_{2} \in R$.

Then the boundary value problem (1)-(2) has a unique solution.

Proof: Define the operator $J: C([a, T], R) \rightarrow C([a, T], R)$ by

where

$$
(J y)(t)={ }_{a}^{t} I^{\alpha} f(t, y)+\sum_{i=1}^{n} \frac{k_{i}(t-a)^{\alpha-i}}{\Gamma(\alpha-i+1)}
$$

$$
k_{i}=\frac{1}{\beta_{i}+\gamma_{i}}\left[C_{i}-\gamma_{i} \quad{ }_{a}^{T} I^{i} f(t, y)-\gamma_{i} \sum_{m=1}^{i-1} \frac{k_{m}(T-a)^{i-m}}{\Gamma(i-m+1)}\right] .
$$

We have to show that $\mathrm{J}$ has a fixed point on $B_{r}$ which will be the solution of (1)-(2). First of all, we need to show that $J B_{r} \subset B_{r}$, where $B_{r}=\{y \in C$ : $\|y\| \leq r\}$. For $y \in B_{r}$, we have

$$
\|(J y)(t)\| \leq \frac{1}{\Gamma(\alpha)} \int_{a}^{t}(t-s)^{\alpha-1}\|f(s, y)\| d s+\sum_{i=1}^{n} \frac{\left\|k_{i}\right\|(t-a)^{\alpha-i}}{\Gamma(\alpha-i+1)},
$$

now, we need to find $\left\|k_{i}\right\|$ as follows

$$
\left\|k_{i}\right\| \leq \frac{1}{\beta_{i}+\gamma_{i}}\left[C_{i}+\gamma_{i} \quad{ }_{a}^{T} I^{i}\|f(t, y)\|+\gamma_{i} \sum_{m=1}^{i-1} \frac{\left\|k_{m}\right\|(T-a)^{i-m}}{\Gamma(i-m+1)}\right]
$$

when $i=1$ and by using $\left(H_{1}\right)$, we obtain $\left\|k_{1}\right\| \leq \frac{1}{\beta_{1}+\gamma_{1}}\left[C_{1}+M \gamma_{1}(T-a)\right]=p_{1}$, 
when $i=2$, we have $\left\|k_{2}\right\| \leq \frac{1}{\beta_{2}+\gamma_{2}}\left[C_{2}+M \gamma_{2} \frac{(T-a)^{2}}{2}+\gamma_{2} p_{1}(T-a)=p_{2}\right.$, by the same way, when $i=n$, we obtain

$$
\begin{aligned}
\left\|k_{n}\right\| \leq & \frac{1}{\beta_{n}+\gamma_{n}}\left[C_{n}+\gamma_{n} M \frac{(T-a)^{n}}{(n !)}+\gamma n \frac{p_{1}(T-a)^{n-1}}{\Gamma(n)}+\gamma_{n} \frac{p_{2}(T-a)^{n-2}}{\Gamma(n-1)}\right. \\
& \left.+\gamma_{n} \frac{p_{3}(T-a)^{n-3}}{\Gamma(n-2)}+\ldots+\gamma_{n} \frac{p_{n-1}(T-a)}{\Gamma(2)}\right]=p_{n} .
\end{aligned}
$$

Thus from equation (11), we have

$$
\|(J y)(t)\| \leq \frac{M T^{\alpha}}{\Gamma(\alpha+1)}+\sum_{i=1}^{n} \frac{p_{i} \theta}{\Gamma(\alpha-i+1)}
$$

where

$$
\theta= \begin{cases}T^{n-i} & \text { when }(t-a) \geq 1 \\ 10 & \text { when } 0.1 \leq(t-a)<1\end{cases}
$$

Now, take $x, y \in C$ and for each $t \in[a, T]$ we obtain

where

$$
\|(J x)(t)-(J y)(t)\| \leq{ }_{a}^{t} I^{\alpha}\|f(t, x)-f(t, y)\|+\sum_{i=1}^{n} \frac{\left\|k_{i}-l_{i}\right\|(t-a)^{\alpha-i}}{\Gamma(\alpha-i+1)},
$$

$$
\begin{aligned}
k_{i} & =\frac{1}{\beta_{i}+\gamma_{i}}\left[\begin{array}{ll}
C_{i}-\gamma_{i} & \quad{ }_{a}^{T} I^{i} f(t, x)-\gamma_{i} \sum_{m=1}^{i-1} \frac{k_{m}(T-a)^{i-m}}{\Gamma(i-m+1)}
\end{array}\right] \\
l_{i} & =\frac{1}{\beta_{i}+\gamma_{i}}\left[\begin{array}{ll}
C_{i}-\gamma_{i} & { }_{a}^{T} I^{i} f(t, y)-\gamma_{i} \sum_{m=1}^{i-1} \frac{l_{m}(T-a)^{i-m}}{\Gamma(i-m+1)}
\end{array}\right] .
\end{aligned}
$$

Now, we have to find $\left\|k_{i}-l_{i}\right\|$ as follows

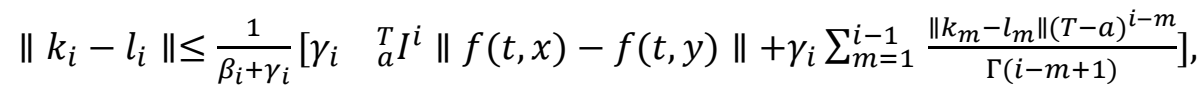

when $i=1$, we have

$$
\left\|k_{1}-l_{1}\right\| \leq q_{1}\|x-y\|, \quad \text { and } q_{1}=\frac{Q}{\beta_{1}+\gamma_{1}}\left[\gamma_{1}(T-a)\right]
$$

when $i=2$, we have

$$
\left\|k_{2}-l_{2}\right\| \leq q_{2}\|x-y\|, \quad \text { and } q_{2}=\frac{1}{\beta_{2}+\gamma_{2}}\left[\gamma_{2} Q \frac{(T-a)^{2}}{2}+\gamma_{2} \frac{q_{1}(T-a)}{\Gamma(2)}\right]
$$

and so on until we find the general form of problem (1)-(2) when $i=n$, we obtain

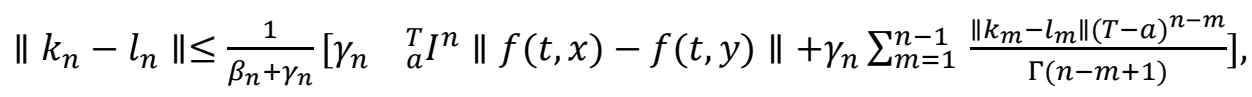




$$
\left\|k_{n}-l_{n}\right\| \leq q_{n}\|x-y\|, \quad \text { and } \quad q_{n}=\frac{\gamma_{n}}{\beta_{n}+\gamma_{n}}\left[Q \frac{(T-a)^{n}}{n !}+\sum_{m=1}^{n-1} \frac{q_{m}(T-a)^{n-m}}{\Gamma(n-m+1)}\right]
$$

Then from equation(12), we get

$$
\begin{aligned}
& \|(J x)(t)-(J y)(t)\| \leq{ }_{a}^{t} I^{\alpha}\|f(t, x)-f(t, y)\|+\sum_{i=1}^{n} \frac{q_{i}\|x-y\|(t-a)^{\alpha-i}}{\Gamma(\alpha-i+1)} \\
& \|(J x)(t)-(J y)(t)\| \leq\left[\frac{Q}{\Gamma(\alpha+1)} T^{\alpha}+\sum_{i=1}^{n} \frac{\theta q_{i}}{\Gamma(\alpha-i+1)}\right]\|x-y\|
\end{aligned}
$$

Since $\left[\frac{Q}{\Gamma(\alpha+1)}(T)^{\alpha}+\sum_{i=1}^{n} \frac{\theta q_{i}}{\Gamma(\alpha-i+1)}\right]<1$, then $J$ is a contraction mapping. Therefore, by using Banach contraction mapping, $J$ has a unique Fixed point which is a unique solution of the problem (1)-(2).

Theorem 3.2 If $\left(H_{1}\right)-\left(H_{2}\right)$ hold with $H_{3}:|f(t, y)| \leq \varphi(t)$,

where $\varphi(t) \in L_{1}(I)$, then the boundary value problem(1)-(2) has at least a solution.

Proof: To prove that the problem (1)-(2) has at least one solution, we need to define two operators $A$ and $B$ that satisfy the three conditions of Krasnosel'skii fixed point theorem.

where

$$
(A x)(t)=\frac{1}{\Gamma(\alpha)} \int_{a}^{t}(t-s)^{\alpha-1} f(s, x(s)) d s, \quad \text { and } \quad(B x)(t)=\sum_{i=1}^{n} \frac{k_{i}(t-a)^{\alpha-i}}{\Gamma(\alpha-i+1)}
$$

$$
k_{i}=\frac{1}{\beta_{i}+\gamma_{i}}\left[C_{i}-\gamma_{i} \quad{ }_{a}^{T} I^{i} f(t, x)-\gamma_{i} \sum_{m=1}^{i-1} \frac{k_{m}(T-a)^{i-m}}{\Gamma(i-m+1)}\right]
$$

Let's show that if $x, y \in B_{r}$, then it is easy to see $A x+B y \in B r$, we have

$$
\|A x(t)+B y(t)\| \leq \frac{T^{\alpha}\|\varphi\|_{L_{1}}}{\Gamma(\alpha+1)}+\sum_{i=1}^{n} \frac{p_{i} \theta}{\Gamma(\alpha-i+1)} .
$$

Next, we can prove that $B x$ is a contraction mapping,

$$
\left\|B x_{1}(t)-B x_{2}(t)\right\| \leq \sum_{i=1}^{n} \frac{\left\|k_{i}-l_{i}\right\|(t-a)^{\alpha-i}}{\Gamma(\alpha-i+1)}
$$

we have that $\left\|k_{i}-l_{i}\right\| \leq q_{n}\left\|x_{1}-x_{2}\right\|$, then equation (13) become

$$
\left\|B x_{1}(t)-B x_{2}(t)\right\| \leq \sum_{i=1}^{n} \frac{q_{n} \theta}{\Gamma(\alpha-i+1)}\left\|x_{1}-x_{2}\right\|
$$

It is clear that $B$ is a contraction mapping because $\sum_{i=1}^{n} \frac{q_{n} \theta}{\Gamma(\alpha-i+1)}<1$. Moreover, $x(t)$ is continuous and this implies that the operator $A x$ is continuous as well.

$$
\|A x(t)\| \leq \frac{1}{\Gamma(\alpha)} \int_{a}^{t}(t-s)^{\alpha-1}\|f(s, y)\| d s \leq \frac{T^{\alpha}\|\varphi\|_{L_{1}}}{\Gamma(\alpha+1)} .
$$

Hence, A is uniformly bounded on $B_{r}$. Next, we prove that the operator $A$ is completely continuous. 
Let $t_{1}, t_{2} \in[a, T], t_{1}<t_{2}$, and $x \in B_{r}$

$$
\begin{gathered}
\left\|A x\left(t_{2}\right)-A x\left(t_{1}\right)\right\| \leq \frac{1}{\Gamma(\alpha)} \int_{a}^{t_{2}}\left(t_{2}-s\right)^{\alpha-1}\|f(s, x(s))\| d s+\frac{1}{\Gamma(\alpha)} \int_{a}^{t_{1}}\left(t_{1}-s\right)^{\alpha-1}\|f(s, x(s))\| d s \\
\left\|A x\left(t_{2}\right)-A x\left(t_{1}\right)\right\| \leq \frac{M_{1}}{\Gamma(\alpha+1)}\left[\left(t_{2}-a\right)^{\alpha}+\left(t_{1}-a\right)^{\alpha}\right] .
\end{gathered}
$$

And this shows that A is relatively compact. And on the other hand By Arzela-Ascoli theorem, A is compact and this concludes the result of Krasnosel'skii theorem by fulfilling the three conditions.

\section{Example I}

In this section, we applying the procedure of theorems (3.1)-(3.2) for the problem (1)-(2), when $1<$ $\alpha \leq 2$. Firstly, recall problem (1)-(2) as

$$
\begin{gathered}
D^{\alpha} y(t)=f(t, y), 1<\alpha \leq 2, \\
\left.\beta_{1} y^{(\alpha-1)}(t)\right|_{t=0}+\gamma_{1} y^{(\alpha-1)}(T)=C_{1}, \\
\left.\beta_{2} y^{(\alpha-2)}(t)\right|_{t=0}+\gamma_{2} y^{(\alpha-2)}(T)=C_{2} .
\end{gathered}
$$

The solution of the fractional boundary value problem (14)-(15) is given by the following steps. By using the lemma (2.3), we can reduce (14) to an equivalent equation

$$
y(t)={ }_{0}^{t} I^{\alpha} f(t, y)+\frac{k_{1} t^{\alpha-1}}{\Gamma(\alpha)}+\frac{k_{2} t^{\alpha-2}}{\Gamma(\alpha-1)}
$$

and then applying the boundary condition (14), we find

$$
\begin{gathered}
k_{1}=\frac{C_{1}-\gamma_{1} \int_{0}^{T} f(s, y) d s}{\left(\beta_{1}+\gamma_{1}\right)} \\
k_{2}=\frac{1}{\left(\beta_{2}+\gamma_{2}\right)}\left[C_{2}-\gamma_{2} T\left(\frac{C_{1}-\gamma_{1} \int_{0}^{T} f(s, y) d s}{\left(\beta_{1}+\gamma_{1}\right)}\right)-\frac{\gamma_{2}}{\Gamma(2)} \int_{0}^{T}(T-s) f(s, y) d s\right] .
\end{gathered}
$$

Therefore the solution of (14)-(15) is

$$
\begin{aligned}
& y(t)=\frac{t^{\alpha-1} C_{1}}{\left(\beta_{1}+\gamma_{1}\right) \Gamma(\alpha)}-\frac{t^{\alpha-1} \gamma_{1} \int_{0}^{T} f(s, y(s)) d s}{\left(\beta_{1}+\gamma_{1}\right) \Gamma(\alpha)}+\frac{t^{\alpha-2} C_{2}}{\left(\beta_{2}+\gamma_{2}\right) \Gamma(\alpha-1)} \\
& -\frac{T t^{\alpha-2} \gamma_{2} C_{1}}{\left(\gamma_{1}+\beta_{1}\right)\left(\gamma_{2}+\beta_{2}\right) \Gamma(\alpha-1)}+\frac{T t^{\alpha-2} \gamma_{1} \gamma_{2} \int_{0}^{T} f(s, y) d s}{\left(\gamma_{2}+\beta_{2}\right)\left(\gamma_{1}+\beta_{1}\right) \Gamma(\alpha-1)}-\frac{t^{\alpha-2} \gamma_{2} \int_{0}^{T}(T-s) f(s, y) d s}{\left(\gamma_{2}+\beta_{2}\right) \Gamma(\alpha-1)} \\
& +{ }_{0}^{t} I^{\alpha} f(t, y)
\end{aligned}
$$

Theorem 4.1 Assume that (H1)-(H2) are held. Then the boundary value problem (14)-(15) has a unique solution. 
proof: Define the operator $J: C([a, T], R) \rightarrow C([a, T], R)$ by

$$
(J y)(t)={ }_{0}^{t} I^{\alpha} f(t, y)+\frac{k_{1} t^{\alpha-1}}{\Gamma(\alpha)}+\frac{k_{2} t^{\alpha-2}}{\Gamma(\alpha-1)}
$$

We have to show that $\mathrm{J}$ has a fixed point on $B_{r}$, and this fixed point is then a solution of (14)-(15). Now we show that $J B_{r} \subset B_{r}$, where $B_{r}=\{y \in C:\|y\| \leq r\}$. For $y \in B_{r}$, we have

$$
\begin{gathered}
\|(J y)(t)\| \leq{ }_{0}^{t} I^{\alpha}\|f(t, y)\|+\frac{\left\|k_{1}\right\| t^{\alpha-1}}{\Gamma(\alpha)}+\frac{\left\|k_{2}\right\| t^{\alpha-2}}{\Gamma(\alpha-1)} \\
\|(J y)(t)\| \leq \frac{M T^{\alpha}}{\Gamma(\alpha+1)}+\frac{p_{1} \theta}{\Gamma(\alpha)}+\frac{p_{2} \theta}{\Gamma(\alpha-1)} \quad \text { where } \quad \theta= \begin{cases}T^{n-i} & \text { when } t \geq 1, \quad i=1,2 \\
10 & \text { when } 0.1 \leq t<1 .\end{cases}
\end{gathered}
$$

Now, take $x, y \in C$ and for each $t \in[a, T]$ we have

$$
\begin{gathered}
\|(J x)(t)-(J y)(t)\| \leq\left(\frac{Q T^{\alpha}}{\Gamma(\alpha+1)}\|x-y\|+\frac{\left\|k_{1}-l_{1}\right\| t^{\alpha-1}}{\Gamma(\alpha)}+\frac{\left\|k_{2}-l_{2}\right\| t^{\alpha-2}}{\Gamma(\alpha-1)}\right), \\
\|(J x)(t)-(J y)(t)\| \leq \omega\|x-y\|
\end{gathered}
$$

where

$$
\omega=\left(\frac{Q T^{\alpha}}{\Gamma(\alpha+1)}+\frac{q_{2} \theta}{\Gamma(\alpha-1)}+\frac{q_{1} \theta}{\Gamma(\alpha)}\right)
$$

Thus, we have proved the uniqueness of the solution of the problem (10)-(11) as $J$ has a unique fixed point because of $\omega<1$.

Theorem 4.2 Assume that $\left(H_{1}\right)-\left(H_{2}\right)$ are held with $\left(H_{3}\right):|f(t, y)| \leq \varphi(t)$, where $\varphi(t) \in L_{1}(I)$.

Then there is at least one solution for the boundary value problem(14)-(15).

Proof: By the same procedure of theorem (3.2), we have two operators

$$
(A x)(t)=\frac{1}{\Gamma(\alpha)} \int_{a}^{t}(t-s)^{\alpha-1} f(s, x(s)) d s, \quad \text { and } \quad(B x)(t)=\frac{k_{1} t^{\alpha-1}}{\Gamma(\alpha)}+\frac{k_{2} t^{\alpha-2}}{\Gamma(\alpha-1)}
$$

Now to show that $A x+B y \in B r$, we have

$$
\|A x(t)+B y(t)\| \leq \frac{T^{\alpha}\|\varphi\|_{L_{1}}}{\Gamma(\alpha+1)}+\frac{p_{1} \theta}{\Gamma(\alpha)}+\frac{p_{2} \theta}{\Gamma(\alpha-1)} .
$$

Now, we need to show that $B x$ is a contraction mapping 


$$
\left\|B x_{1}(t)-B x_{2}(t)\right\| \leq\left(\frac{q_{1} \theta}{\Gamma(\alpha)}+\frac{q_{2} \theta}{\Gamma(\alpha-1)}\right)\left\|x_{1}-x_{2}\right\|
$$

Since $\frac{q_{1} \theta}{\Gamma(\alpha)}+\frac{q_{2} \theta}{\Gamma(\alpha-1)}<1$, then $B$ is a contraction mapping. Moreover, continuity of $x(t)$ implies that the operator $A x$ is continuous

$$
\|A x(t)\| \leq \frac{T^{\alpha}\|\varphi\|_{L_{1}}}{\Gamma(\alpha+1)}
$$

Hence, A is uniformly bounded on $B_{r}$. Now we prove that the operator $A$ is completely continuous. Let $t_{1}, t_{2} \in[a, T], t_{1}<t_{2}$, and $x \in B_{r}$.

$$
\left\|A x\left(t_{2}\right)-A x\left(t_{1}\right)\right\| \leq \frac{M_{1}}{\Gamma(\alpha+1)}\left[\left(t_{2}-a\right)^{\alpha}+\left(t_{1}-a\right)^{\alpha}\right] .
$$

Thus, A is relatively compact. Then, by the results of Arzela-Ascoli theorem, A is compact. And meets the theorems (3.1) -(3.2), then the problem (14)-(15) has at least a solution.

Example1:.Consider the following boundary value problem:

$$
\left\{\begin{array}{l}
D^{\alpha} y(t)=\frac{e^{t}}{(10+t)} y, \quad 0<\alpha \leq 1 \\
y^{(\alpha-1)}(a)+y^{(\alpha-1)}(T)=1
\end{array}\right.
$$

(1)

$$
\begin{aligned}
& \text { Let } a=0, T=1, \alpha=\frac{1}{2}, \beta_{1}=1, \gamma_{1}=1 \text {, by using }(\mathrm{H} 2) \text {, we find } \\
& \qquad \begin{array}{c}
|f(t, x)-f(t, y)| \leq \frac{1}{10}|x-y|, \quad Q=\frac{1}{10} \quad \text { and } \quad q_{1}=\frac{Q \gamma_{1} T}{\beta_{1}+\gamma_{1}}=\frac{1}{20} \\
\text { and } \quad \theta=10, \quad \text { when } \quad 0.1 \leq t<1 \quad \text { wehave } \\
\omega=\frac{Q}{\Gamma(\alpha+1)} T^{\alpha}+\frac{\theta q_{1}}{\Gamma(\alpha)}=\frac{1}{20 \Gamma(3 / 2)}+\frac{\theta}{20 \Gamma(1 / 2)}
\end{array}
\end{aligned}
$$

we find $\omega=0.338445644$. The boundary value problem (16) has a unique solution.

(2) Let $\alpha=\frac{1}{2}, a=1, T=2, \beta_{1}=1, \gamma_{1}=1$, by using (H2), we have

$$
\begin{gathered}
|f(t, x)-f(t, y)| \leq 0.2471165|x-y| \Rightarrow Q=0.2471165 \\
q_{1}=\frac{Q \gamma_{1}(T-1)}{\beta_{1}+\gamma_{1}}=0.12355825 \\
\text { and } \omega=\frac{Q}{\Gamma(\alpha+1)} T^{\alpha}+\frac{\theta q_{1}}{\Gamma(\alpha)}=\frac{0.2471165(2)^{\frac{1}{2}}}{\Gamma(3 / 2)}+\frac{0.12355825 \theta}{\Gamma(1 / 2)}
\end{gathered}
$$

from the condition $\theta=T^{n-i}$, when $(t-1) \geq 1$, we find $\omega=0.463957782$. Then the boundary value problem (16) has a unique solution. 
Example2:.Consider the following boundary value problem:

$$
\left\{\begin{array}{l}
D^{\alpha} y(t)=\frac{e^{t}|y|}{\left(9+e^{t}\right)(1+|y|)}, \quad 1<\alpha \leq 2 \\
y^{(\alpha-1)}(a)+y^{(\alpha-1)}(T)=1 \\
y^{(\alpha-2)}(a)+y^{(\alpha-2)}(T)=1 .
\end{array}\right.
$$

(1) Let $a=0, T=1, \alpha=\frac{3}{2}, \gamma_{1}=\gamma_{2}=1, \beta_{1}=\beta_{2}=1$, using (H2), we have

$$
\begin{gathered}
|f(t, x)-f(t, y)| \leq \frac{1}{10}|x-y|, \quad Q=\frac{1}{10}, \quad q_{1}=\frac{Q \gamma_{1} T}{\beta_{1}+\gamma_{1}}=\frac{1}{20}, \\
\text { and } q_{2}=\frac{1}{\beta_{2}+\gamma_{2}}\left(\gamma_{2} Q \frac{T^{2}}{2}+\gamma_{2} \frac{q_{1} T}{\Gamma(2)}\right)=\frac{1}{20} \\
\omega=\frac{Q T^{\alpha}}{\Gamma(\alpha+1)}+\frac{q_{1} \theta}{\Gamma(\alpha)}+\frac{q_{2} \theta}{\Gamma(\alpha-1)}=\frac{1}{10 \Gamma(5 / 2)}+\frac{\theta}{20 \Gamma(3 / 2)}+\frac{\theta}{20 \Gamma(1 / 2)},
\end{gathered}
$$

from the condition $\theta=10$, when $0.1 \leq t<1$, then $\omega=0.921324354$, and problem (17) has a unique solution.

(2) Let $\alpha=\frac{3}{2}, \gamma_{1}=\gamma_{2}=1, \beta_{1}=\beta_{2}=1, a=1, T=2$, from the problem (17) and using (H2), we obtain

$$
\begin{gathered}
|f(t, x)-f(t, y)| \leq \frac{e^{t}}{\left(9+e^{t}\right)}|x-y| \leq 0.231969316|x-y| \Leftrightarrow Q=0.231969316 \\
q_{1}=\frac{Q \gamma_{1}(T-a)}{\beta_{1}+\gamma_{1}}=0.115984658 \quad q_{2}=\frac{1}{\beta_{2}+\gamma_{2}}\left(\frac{\gamma_{2} Q(T-a)^{2}}{2}+\frac{\gamma_{2} q_{1}(T-a)}{\Gamma(2)}\right)=0.115984658 \\
\omega=\frac{Q T^{\alpha}}{\Gamma(\alpha+1)}+\frac{q_{2} \theta}{\Gamma(\alpha-1)}+\frac{q_{1} \theta}{\Gamma(\alpha)}=\frac{0.231969316(2)^{3 / 2}}{\Gamma(5 / 2)}+\frac{0.115984658 \theta}{\Gamma(3 / 2)}+\frac{0.115984658 \theta}{\Gamma(1 / 2)}
\end{gathered}
$$

from the condition $\theta=T^{n-i}$, when $(t-1) \geq 1$, we find $\omega=0.820580848$ Therefore, by Theorem 3.1, the boundary value problem (17) has a unique solution. 


\section{REFERENCES}

Afshari H., Marasi H., and Aydi H., Existence and Uniqueness of Positive Solutions for Boundary Value Problems of Fractional Differential Equations, Filomat, 31(9),(2017), 2675-2682.

Arora H.L. and J. G Al-Shamani, Stability of differential equation of non integer order through fixed point in the large, Indian J. pure ,appl. Math.,11(3)(1980),307-313.

Barrett J.H., Differential Equation of non-integer order, canad. J. Math.,6(4)(1954),529-541.

Benchora M., J. Henderson, S.K. Natouyas, A. Ouahab, Existence results for fractional order functional differential equations with infinite delay, J. Math. Anal. Appl., 338 (2008), pp. 1340-1350.

Dumitru Baleanu, Sayyedeh Zahra Nazemi, Shahram Rezapour, Existence and uniqueness of solutions for multi-term nonlinear fractional integro-differential equations, Advances in Difference Equations, Advances in Difference Equations 2013, 2013:368, 2-17.

[6] Kilbas A.A., H.M. Srivastava, J.J. Trujillo, Theory and Applications of Fractional Differential Equations, Elsevier Science B.V, Amsterdam (2006).

Krasnosel'skii M. A. , "Two remarks on the method of successive approximations," Uspekhi Matematicheskikh Nauk, vol. 10, no. 1(63), pp. 123-127,(1955).

Lakshmikanthama V., A.S.Vatsalab, Basic theory of fractional differential equations, -
Nonlinear Analysis: Theory, Methods and Applications, Volume 69, Issue 8, 15 October 2008, pp. 2677-2682.

Liu Yuji. "Existence of Unbounded Positive Solutions of Boundary Value Problems for Differential Systems on Whole Lines." Filomat, vol. 30, no. 13, 2016, pp. 35473564.

Oldham, K.B. and Spanier, J., The Fractional Calculus, Academic Press, New York, London(1974).

Podlubny I., Fractional differential equation, Mathematics in Science and Engineering. Academic Press, San Diego(1999).

Rabha W Ibrahim, A Kılıçman, FH Damag, Existence and uniqueness for a class of iterative fractional differential equations, Advances in Difference Equations 2015 (1), 78 .

Rian Yan, Shurong Sun, Ying Sun, Zhenlai Han, Boundary value problems for fractional differential equations with nonlocal boundary conditions, Advances in Difference Equations, 2013, 2013:176,212.

Shaher M. Momani, "Local and global existence theorem on fractional integro-differential equations", Journal of Fractional Calculus, Vol. 18, pp.81-86, (2000).

Shayma Adil Murad, Samir Hadid "An existence and uniqueness theorem for fractional differential equation with integral boundary condition", J. Frac. Calc. Appl 3 
(6),2012, 1-9.

Shayma A. Murad, Hussein J.Zekri and Samir

Hadid, "Existence and Uniqueness

Theorem of Fractional Mixed Volterra-

Fredholm Integrodifferential Equation

with Integral Boundary Conditions",

International Journal of Differential

Equations , Volume 2011, Article ID 304570, 15 pages.

Shuman Meng and Yujun Cui1, The Uniqueness

Theorem of the Solution for a Class of
Differential Systems with Coupled Integral

Boundary Conditions", Discrete Dynamics in Nature and Society, Volume 2018, Article ID 9601868, 7 pages. https://doi.org/10.1155/2018/9601868.

Yongqing Wang, Existence of Uniqueness and Nonexistence Results of Positive Solution for Fractional Differential Equations Integral Boundary Value Problems, Journal of Function Spaces, Volume 2018, Article ID 1547293, 7 pages. 